\title{
Is India Ready to Address its Biggest Public Health Challenge? Forecasts from the Publicly Available Data on COVID-19
}

\author{
Ramesh Athe, Rinshu Dwivedi*, Nagapraveen Modem
}

\begin{abstract}
Background: The COVID-19 pandemic in India has become the biggest public health challenge. If we go by the number of confirmed cases and casualties, the situation seems to be a matter of grave concern. The lock-down measures and social-distancing practices have played an important role in impeding the spread of COVID-19. However, accurate forecasting is required to prepare the healthcare-system for future plan-of-action. Objectives: The present study aims to predict the trends in the outbreak of COVID-19 in India, by forecasting on the basis of publicly-available data. Methods: The data has been obtained from https://www. covid19india.org, https://www.worldometers.info/coronavirus and ICMR reported publiclyavailable information on COVID-19. The number of confirmed cases are growing exponentially as per the real-time-data. For forecasting the trends in terms of confirmed, active, recovered, and death cases by using the autoregressive-integrated-moving-averages (ARIMA) model. Results: Findings reveal the estimated value of the point-forecast for total confirmed, active, recovered, and death cases. Estimates indicate that the total confirmed cases were increasing at the rate of $3.48 \%$, active cases at $2.92 \%$, recovered cases at $4.09 \%$ and death cases at the rate of $3.51 \%$ daily across the country. It was also observed that the death rate was lower for the states and union territories with a higher detection rate. Conclusion: Substantial public health interventions were implemented immediately by the Government of India to control the spread of COVID-19. Due to the shortage of healthcare resources in the country, early detection is imperative along with accurate forecasting. It will help in reducing the associated cost of acute care for the majority of infected cases and unnecessary burden on the healthcare system.

Key words: Coronavirus Disease (COVID-19), Forecasting, ARIMA, Public-health, Emergency-preparedness, India.
\end{abstract}

\section{Ramesh Athe, Rinshu Dwivedi*, Nagapraveen Modem \\ Indian Institute of Information Technology, Dharwad, Karnataka, INDIA. \\ Correspondence \\ Dr. Rinshu Dwivedi \\ Assistant Professor, Indian Institute of Information Technology, Trichy-620015, Tamil Nadu, INDIA \\ Mobile no: +917683981979 \\ Email: rinshudwivedi999@gmail.com \\ History \\ - Submission Date: 21-05-2020 \\ - Revised Date: 16-07-2020 \\ - Accepted Date: 25-08-2020}

DOI : 10.5530/ijmedph.2020.4.47

\section{Article Available online}

http://www.ijmedph.org/v10/i4

\section{Copyright}

(C) 2020 Phcog.Net. This is an openaccess article distributed under the terms of the Creative Commons Attribution 4.0 International license.

\section{INTRODUCTION}

A larger number of individuals and animals have been affected worldwide over the past two decades with three epidemics caused by the family of coronavirus (Severe Acute Respiratory Syndrome (SARS)-2003, Middle East Respiratory Syndrome (MERS)-2012 and Coronavirus Disease (COVID-19). ${ }^{1-3}$ However, significant genetic variations have been recorded among the pathogens of these three epidemics, especially between MERS and COVID-19. Initially the Middle East, Saudi Arabia and China ${ }^{5,6}$ were the hotspots for these epidemics where animals to human transmission cases were recorded. Later, these pathogens were transmitted from human to human in other countries. The outbreak of COVID-19 was first reported from Wuhan, China in December, 2019. ${ }^{7}$ In March, 2020 it was declared as a pandemic by WHO due to huge upsurge in the number of reported cases for COVID-19 as compared to SARS and MERS.

The common symptoms associated with COVD-19 are; fever, tiredness and dry cough. Some people have also experienced symptoms such as nasal congestion, running nose, body pain, sore throat, cough and

diarrhoea, which are quite similar to the common cold conditions. A pooled analysis of 181 confirmed cases of COVID- $19^{8}$ estimated the mean incubation period to be between 5.1 to 11.5 days. The majority of COVID-19 patients have a self-limiting infection and they do recover over time. However, in severe cases, about $10 \%$ of these infected cases may require admission in the intensive care units (ICUs) and some of them may also die.

The elderly population, people with lower immunity and with prior medical conditions are more prone to COVID-19. As per WHO, the majority of the casualties have occurred among the older age population, or people with at least one comorbidity; particularly cardiovascular diseases/hypertension, diabetes and other chronic underlying conditions. Though the other segment of the population is not invincible and people who are practicing even social distancing ( 1 meter or 3 feet) or self-quarantine need to follow a healthy lifestyle. Practicing good sanitary habits such as washing the hands frequently, avoiding repeated contact with face, nose and mouth can

Cite this article : Athe R, Dwivedi R, Modem N. Is India Ready to Address its Biggest Public Health Challenge? Forecasts from the Publicly Available Data on COVID-19. Int J Med Public Health. 2020;10(4):226-30. 
Athe, et al:: Forecasts from the Publically Available Case Data on the Coronavirus Disease (COVID-19)

also help in avoiding the infection. There are two important stages of COVID-19 i.e. stage II and III. In stage II, the transmission takes place due to interaction with infected individuals, while in stage III, there is community spread. ${ }^{9}$

The first case of the COVID-19 in India was reported in January 2020. Being the second-largest populated country with resource constraints, the case detection for COVID-19 was initially low. ${ }^{10,11}$ There were also variations in the predicted estimates of total infected cases. On March 22, 2020, a fourteen hour voluntary public curfew was observed throughout India which was later followed by the lockdown of 75 districts from where any confirmed cases have been reported. The country has introduced the first phase of complete lockdown for 21 days amidst rapidly increasing confirmed cases on March 24, 2020, to control the sustained risk of further spread across the nation. All educational institutions have been closed and tourist visas have been cancelled. All modes of transportation and the majority of commercial establishments have been also shut down to avoid the spread. However, the country has further extended the lockdowns for the second and third phases as well.

As per the available data from the Ministry of Health and Family Welfare (MoHFW), the country has a total of 47480 confirmed cases, along with 24385 recoveries and 2415 deaths on May 13, 2020 (MoHFW COVID-19). ${ }^{12-14}$ According to real-time data, confirmed COVID-19 cases are growing exponentially. As our healthcare system is preparing itself for its biggest battle to treat the infected cases, accurate forecasting's can guide us to prepare the future strategies to manage the existing and expected number of cases. This forecasting dissemination can play a crucial role in briefing the governments and healthcare professionals regarding the measures to implement for better adherence among the public. The publicly available data has been used for the present study to estimate the predicted cases across the country by using the autoregressive-integrated-moving-averages (ARIMA) model. We have assumed that publically available daily case data is legitimate, except reporting biases if any. The objective is not to attempt for meticulous precision but to merely provide useful insights on the COVID-19 scenario in India.

\section{MATERIALS AND METHODS}

Present study has drawn the data from publicly available secondary sources (https://www.covid19india.org; https://www.worldometers.info/ coronavirus/; Ministry of Health and Family Welfare (MoHFW) and Indian Council of Medical Research (ICMR) reports) on COVID-19 confirmed, active, recovered and death cases. ${ }^{12-14}$ Based on the available literature, the average duration between the onset of symptoms and in the case where no recovery takes place, the death may occur in 14 days. ${ }^{15}$ Data was collected and analyzed from inception to May 9, 2020, by using the ARIMA model. This model is one of the popular statistical methods for time-series forecasting. ${ }^{16}$ The model uses the weighted averages of most recently-existing past values in the series to forecast the new values. However, as the observations get older (in time), the importance of forecasted values gets exponentially smaller. A univariate time-series model has been used to forecast the daily confirmed, active, recovered, and death cases in India from May 10 to May 19, 2020. The pattern of forecasted regressive curves were consistent with the actual values, hence the fitted ARIMA model was acceptable for the non-seasonal data. ${ }^{1718}$

Steps to implement ARIMA are: first loading the data and pre-processing it, along with making the time series stationary. Later, we determine the $\mathrm{d}$-value and create an auto-correlation function (ACF) and partial autocorrelation function (PACF) plots. After determining the $\mathrm{p}$ and $\mathrm{q}$ values, we fit the ARIMA model. Lastly, we predict the values on the validation set and calculate the root mean square error (RMSE). However, ARIMA makes the task easier as it eliminates the steps from 6 to 3, which were time-consuming. Steps for auto ARIMA are: Loading the data and preprocessing it to fit the ARIMA model. Then we predict the values on the validation set and calculate the RMSE.

The auto-regressive (AR) component ' $p$ ' represents the number of lags used in the previous values of the regression equation for series $\mathrm{Y}$. The equation for the model is:

$Y_{t}=c+\varnothing_{1} Y_{t-1}+\varnothing_{2} Y_{t-2}+e_{t}$

Where the parameters of the model are $\varnothing_{1}$ and $\varnothing_{2}$.

The model components are given as follows where ' $\mathrm{d}$ ' represents the degree of differencing that is, $\mathrm{d}$ times differencing its current and previous values in the series to stabilize when the assumption of stationary is not fulfilled. A moving average (MA) is represented by ' $q$ ' which indicates the order of number of terms included in the model and estimates the model error by combining the previous error term $(\mathrm{s}) e_{t}$.

$Y_{t}=c+\theta_{1} e_{t-1}+\theta_{2} e_{t-2}+\ldots+\theta_{q} e_{t-q}+e_{t}$

Non-seasonal ARIMA model components such as differencing, moving averages and auto-regressive lags can be rewritten in the form of linear equation as:

$Y_{d}=c+\varnothing_{1} e_{d t-1}+\varnothing_{2} e_{d t-2}+\ldots+\theta_{1} e_{t-1}+\varnothing_{q} e_{t-q}+e_{t}$

Where $Y_{d}$ is $Y$-differenced ' $d$ 'times and ' $c$ ' is constant.

However certain limitations are associated with the ARIMA models, such as its dependency on existing series of values to forecast the projected constant series of estimated values, which requires a long and stable series of data. Also, these models merely explain the approximated historical patterns rather than providing any interpretation for the underlying structure of data mechanisms. The $\mathrm{R}$ software (ARIMA module) was used to analyze the data. ${ }^{19}$

\section{RESULTS}

Estimates from the present study reports that the total number of COVID-19 cases in India could be approximately 94682 (95\% CI: $87362.22,102000.46)$ as on May 19, 2020. The predicted confirmed and active cases from May 10 to May 19, 2020, are presented in Table 1. The total confirmed cases will be increasing approximately at the rate of $3.48 \%$ and active cases at the rate of $2.92 \%(62975 ; 95 \%$ CI: $55099.38,70852.12)$ daily, due to COVID-19. For recovered cases, the rate of increase will be at $4.09 \%(31774 ; 95 \% \mathrm{CI}: 28834.37,34714.30)$ and for death cases, it will increase at $3.51 \%$ (3183; 95\% CI: 2852.86, 3513.44) (Table 2).

The number of confirmed and active cases of COVID-19 are depicted by Figure 1(a) and 1(b). The red colour curve indicates the cases from March 11,2020, to May 9, 2020, based on the actual data for the confirmed and active cases. This data has been used as a base for predicting the number of confirmed and active cases in India, which was portrayed via the blue colour trend line i.e. predicted region. It is predicting the number of confirmed and active cases from May 10 to May 19, 2020 in India. As per the predictions, the count of confirmed and active cases in India will fall in the predicted region. Here, Y-axis represents the number of confirmed cases and X-axis represents the days from March 11, 2020, to May 19, 2020. Similarly, recovered and death cases were depicted in Figure 2(a) and 2(b).

\section{DISCUSSION}

The COVID-19 pandemic in India has become a public health threat and as per the forecasts, the situation seems to be a matter of serious concern. This novel virus represents a tremendous challenge for the public health scenario in India, as neither, we have a vaccine nor we have a medication to treat this virus. The present study emphasizes that accuracy in the forecasting's will not only help in timely detection and required care, 
Table 1: Forecasting estimates of confirmed and active cases from the ARIMA method.

\begin{tabular}{lcccccc} 
Date & \multicolumn{3}{c}{ Confirmed cases } & \multicolumn{3}{c}{ Active cases } \\
& $\begin{array}{c}\text { Point } \\
\text { estimate }\end{array}$ & 95\% confidence intervals & $\begin{array}{c}\text { Point } \\
\text { estimate }\end{array}$ & 95\% confidence intervals \\
\hline 10 May 2020 & 66025.82 & 65506.97 & 66544.66 & 46874.00 & 45568.22 & 48179.78 \\
11 May 2020 & 69196.07 & 68240.64 & 70151.50 & 48637.84 & 46180.50 & 50465.17 \\
12 May 2020 & 72387.91 & 70859.85 & 73915.96 & 50445.14 & 48018.32 & 52871.96 \\
13 May 2020 & 75569.95 & 73404.54 & 77735.36 & 52226.32 & 49157.41 & 55295.23 \\
14 May 2020 & 78756.44 & 75876.91 & 81635.96 & 54023.20 & 50257.46 & 57788.95 \\
15 May 2020 & 81940.91 & 78285.43 & 85596.39 & 55810.65 & 51305.59 & 60315.70 \\
16 May 2020 & 85126.29 & 80634.58 & 89618.01 & 57603.76 & 52314.58 & 62892.94 \\
17 May 2020 & 88311.27 & 82928.39 & 93694.14 & 59393.47 & 53280.44 & 65506.50 \\
18 May 2020 & 91496.42 & 85170.14 & 97822.71 & 61185.22 & 54208.93 & 68161.52 \\
19 May 2020 & 94681.50 & 87362.53 & 102000.46 & 62975.75 & 55099.38 & 70852.12
\end{tabular}

Table 2: Forecasting estimates of recovered and death cases from the ARIMA method.

\begin{tabular}{|c|c|c|c|c|c|c|}
\hline \multirow[t]{2}{*}{ Date } & \multicolumn{3}{|c|}{ Recovered cases } & \multicolumn{3}{|c|}{ Death cases } \\
\hline & \multirow{2}{*}{$\begin{array}{c}\text { Point } \\
\text { estimate } \\
20531.92\end{array}$} & \multicolumn{2}{|c|}{$95 \%$ confidence intervals } & \multirow{2}{*}{$\begin{array}{c}\text { Point } \\
\text { estimate } \\
2209.21\end{array}$} & \multicolumn{2}{|c|}{$95 \%$ confidence intervals } \\
\hline 10 May 2020 & & 20352.03 & 20711.81 & & 2176.74 & 2241.69 \\
\hline 11 May 2020 & 21781.07 & 21486.31 & 22075.84 & 2317.43 & 2260.60 & 2374.26 \\
\hline 12 May 2020 & 23030.23 & 22536.55 & 23523.92 & 2425.65 & 2342.42 & 2508.87 \\
\hline 13 May 2020 & 24279.39 & 23533.94 & 25024.84 & 2533.86 & 2421.86 & 2645.86 \\
\hline 14 May 2020 & 25528.55 & 24491.42 & 26565.67 & 2642.08 & 2498.94 & 2785.21 \\
\hline 15 May 2020 & 26777.70 & 25415.23 & 28140.17 & 2750.29 & 2573.78 & 2926.79 \\
\hline 16 May 2020 & 28026.02 & 26309.12 & 29744.60 & 2858.51 & 2646.50 & 3070.51 \\
\hline 17 May 2020 & 29276.02 & 27175.70 & 31376.34 & 2966.72 & 2717.19 & 3216.25 \\
\hline 18 May 2020 & 30525.18 & 28016.92 & 33033.43 & 3074.93 & 2785.95 & 3363.92 \\
\hline 19 May 2020 & 31774.33 & 28834.37 & 34714.30 & 3183.15 & 2852.86 & 3513.44 \\
\hline
\end{tabular}

recovered cases forecast from ARIMA $(1,2,0)$

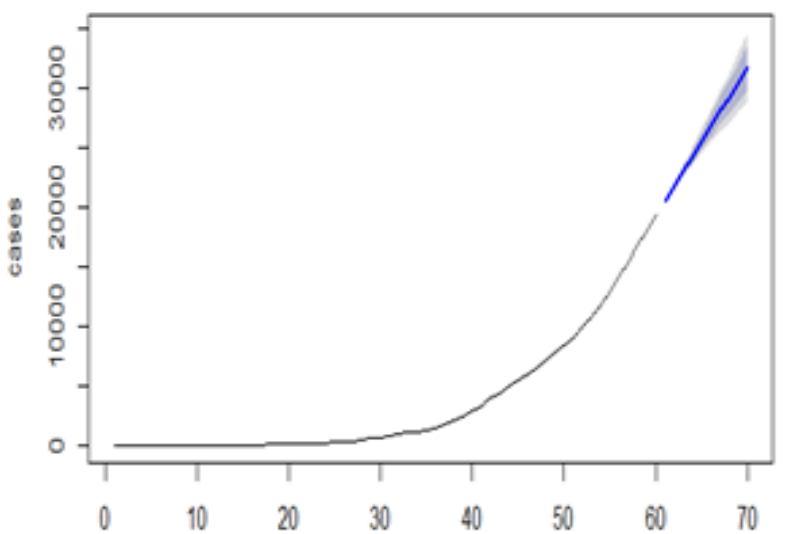

(a) Recovered cases

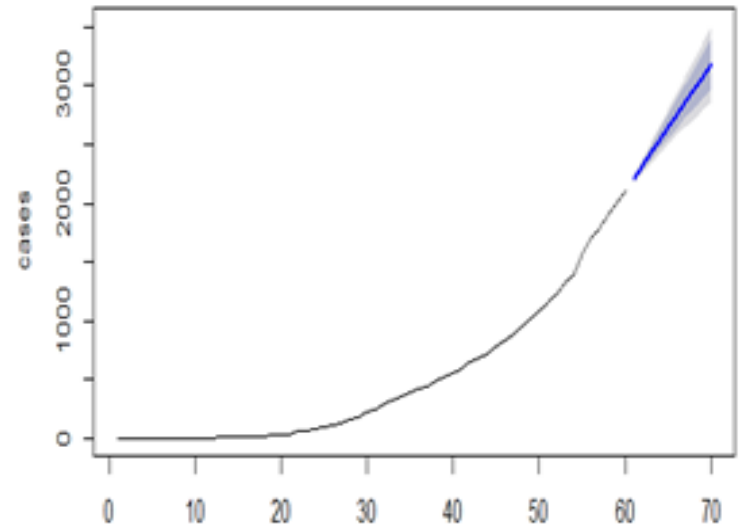

(b) Death cases

Figure 1: Forecasting estimates of recovered and death cases through the ARIMA method.

but also to reduce the associated cost of providing critical care to these patients and overcrowding of the limited healthcare resources.

The estimated forecasts from the present study have been cross-verified with the actual cases reported on May 10-11, 2020 and they were approximately tallying with actual Figures with some minor variation. These estimates may guide us for improved and high detection rates, which may enhance the testing capacity of the country which is essentially required. Late detection may put the cases in a greater need for 
Confirmed cases forecast trom ARIMA(1,2,0)

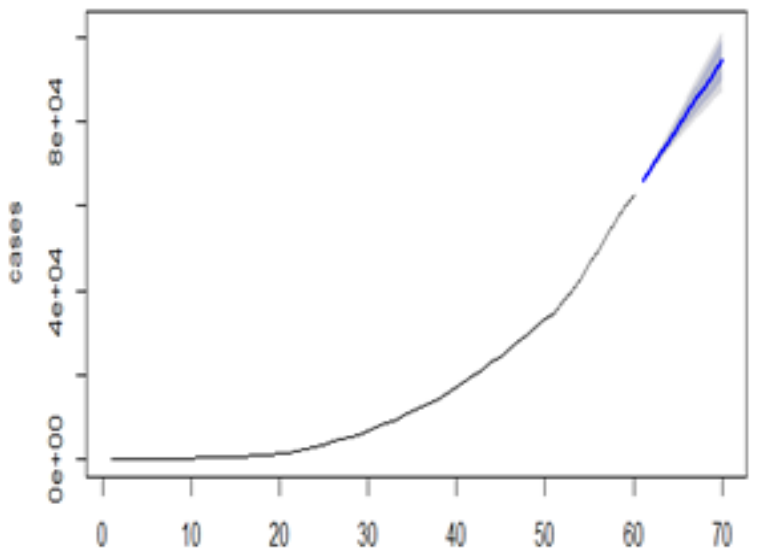

(a)Confirmed cases active cases forecast from ARIMA(1,2,0)

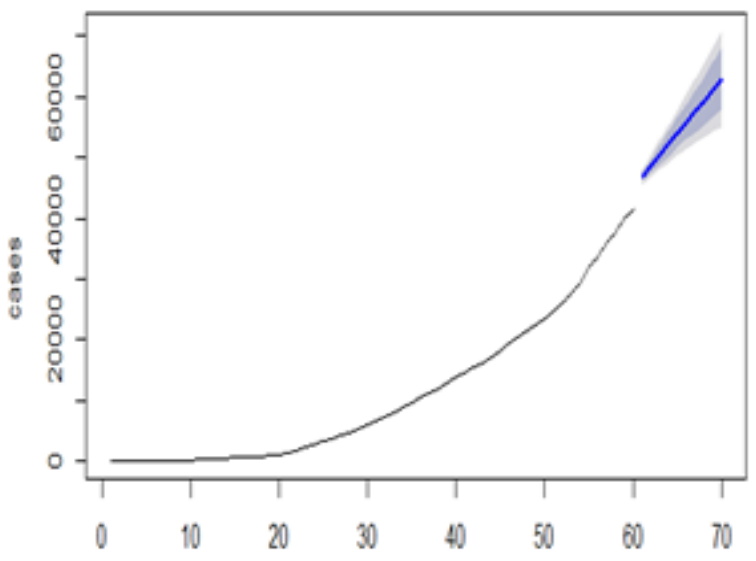

(b) Active cases

Figure 2: Forecasting estimates of confirmed and active cases through the ARIMA method.

ventilation and ICU care, which imposes a significant economic burden on the healthcare system. ${ }^{20}$ It was also observed that there is a need for the study based on the clinical characteristics of the deceased due to COVID-19 to help the epidemiologists and public health practitioners to address the concerns more effectively. As per the available literature, the health care workers have also reported contracting the disease and some have died also. These people are working in challenging conditions, with fewer resources. ${ }^{21,22}$ They need to be supported with adequate training and resources, therefore the preparations of the health facilities, equipped with adequate resources are a must for creating a conducive environment for health professionals to work efficiently. These challenges can be addressed to a large extent if we can estimate the growth of future cases accurately by forecasting. ${ }^{23}$ It will not only help the health professional but also strengthen the readiness of the healthcare system and administrative officials to make suitable decisions.

Rapid assessments and clinical trials are also critical to control the risk and to ensure efficient utilization of the available resources. No doubt, substantial public health interventions have been immediately implemented by the Government of India; there is a need for improved preparedness plans and mitigation strategies for rapid deployment. The delay in required actions often converts a manageable health emergency condition into a miserably overburdened healthcare system. ${ }^{24,25}$ Ultimately, people have to understand the severity of the disease and respect these restrictions implemented by the government in terms of lockdown and other restrictions to help in reducing the growth of further infections. As the spread of the disease is getting severe, it's important to protect yourself and your families and most importantly your community as an act of solidarity.

\section{ACKNOWLEDGEMENT}

Authors Acknowledge the Government of India, open data sources and others through which this study became possible.

\section{CONFLICT OF INTEREST}

All the authors declare that they have no conflict of interest.

\section{Author's contribution}

RA and RD conceptualized the study; data has been collected by RA and PKM. Analysis has been carried out by RA and PKM. The manuscript was written by the RD and RA. All the authors have read and approved the manuscript.

\section{REFERENCES}

1. Yang $Y$, Peng F, Wang R, Guan $K$, Jiang $T, X u$ G, et al. The deadly coronaviruses: The 2003 SARS pandemic and the 2020 novel coronavirus epidemic in China. Journal of Autoimmunity. 2020;102434.

2. Kerkhove MDV, Peiris MJ, Malik MR, Embarek BP. Interpreting results from environmental contamination studies of Middle East respiratory syndrome coronavirus. Clinical Infectious Diseases. 2016;63(8):1142.

3. Hilgenfeld R, Peiris M. From SARS to MERS: 10 years of research on highly pathogenic human coronaviruses. Antiviral Research. 2013;100(1):286-95.

4. Kolifarhood G, Aghaali M, Saadati HM, Taherpour N, Rahimi S, Izadi N, et al. Epidemiological and clinical aspects of Covid-19: A narrative review. Archives of Academic Emergency Medicine. 2020;8(1).

5. Sutton TC, Subbarao K. Development of animal models against emerging coronaviruses: From SARS to MERS coronavirus. Virology. 2015;479:247-58.

6. Wu T, Perrings C, Kinzig A, Collins JP, Minteer BA, Daszak P. Economic growth, urbanization, globalization and the risks of emerging infectious diseases in China: A review. Ambio. 2017;46(1):18-29.

7. Wu Z, McGoogan JM. Characteristics of and important lessons from the coronavirus disease 2019 (COVID-19) outbreak in China: Summary of a report of 72314 cases from the Chinese Center for Disease Control and Prevention. Jama. 2020;323(13):1239-42.

8. Lauer SA, Grantz KH, Bi Q, Jones FK, Zheng Q, Meredith HR, et al. The incubation period of coronavirus disease 2019 (COVID-19) from publicly reported confirmed cases: Estimation and application. Annals of Internal Medicine. 2020;172(9):577-82.

9. Anderson RM, Heesterbeek H, Klinkenberg D, Hollingsworth TD. How will country-based mitigation measures influence the course of the COVID-19 epidemic?. The Lancet. 2020;395(10228):931-4.

10. Singhal T. A review of coronavirus disease-2019 (COVID-19). The Indian Journal of Pediatrics. 2020;1-6.

11. Dikid T, Jain SK, Sharma A, Kumar A, Narain JP. Emerging and re-emerging infections in India: An overview. The Indian Journal of Medical Research. 2013;138(1):19.

12. Ministry of Health and Family Welfare (MoHFW), COVID-19 India, Government of India. COVID-19; https://www.mohfw.gov.in/

13. Coronavirus Outbreak in India, A crowd sourced initiative. https://www. covid19india.org, 2020.

14. COVID-19 Coronavirus Pandemic. https://www.worldometers.info/

15. Cascella M, Rajnik M, Cuomo A, Dulebohn SC, DiNapoli R. Features, evaluation and treatment coronavirus (COVID-19). In Statpearls. 2020. StatPearls Publishing.

16. Pankratz A. Forecasting with univariate Box-Jenkins models: Concepts and cases. John Wiley and Sons. 2009.

17. Dhanwant JN, Ramanathan V. Forecasting COVID 19 growth in India using the Susceptible-Infected-Recovered (SIR) model. arXiv preprint arXiv:2004.00696. 2020.

18. Bhattacharyya A, Bhowmik D, Mukherjee J. Forecast and interpretation of daily affected people during 21 days lockdown due to COVID 19 pandemic in India. 
Med Rxiv. 2020

19. Hyndman RJ, Khandakar Y. Automatic time series for forecasting: the forecast package for R. Clayton VIC, Australia: Monash University, Department of Econometrics and Business Statistics. 2007.

20. Angarai R, Sharma K. The need to redesign the hospital ICUs for Covid-19 patients to save the medical fraternity.

21. Ferguson N, Laydon D, Nedjati GG, Imai N, Ainslie K, Baguelin M, et al. Report 9: Impact of non-pharmaceutical interventions (NPIs) to reduce COVID19 mortality and healthcare demand.

22. Pandey SK, Sharma V. A tribute to frontline corona warriors-Doctors who sacrificed their life while saving patients during the ongoing COVID-19 pandemic. Indian Journal of Ophthalmology. 2020;68(5):939.

23. Sharma SN Singh SK. Challenges and Threats due to Deadly Corona Virus in India and Dealing it with Social Vaccine (distancing)-the the Only Weapon Journal of Communicable Diseases (E-ISSN: 2581-351X and P-ISSN: 0019 5138). 2020;52(1):7-13.

24. Nadim SS, Ghosh I, Chattopadhyay J. Short-term predictions and prevention strategies for COVID-2019: A model-based study. arXiv preprint arXiv:2003.08150. 2020

25. Sohrabi C, Alsafi Z, O'Neill N, Khan M, Kerwan A, Al-Jabir A, et al. World Health Organization declares global emergency: A review of the 2019 novel coronavirus (COVID-19). International Journal of Surgery. 2020

Cite this article : Athe R, Dwivedi R, Modem N. Is India Ready to Address its Biggest Public Health Challenge? Forecasts from the Publicly Available Data on COVID-19. Int J Med Public Health. 2020;10(4):226-30. 